\title{
MEASURING CHARGE AND CHARGE DECAY IN FLOATING-ELECTRODE ELECTROSTATIC ACTUATORS

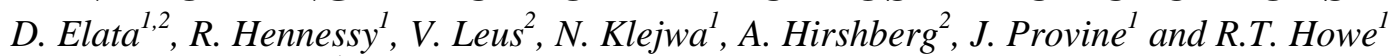 \\ ${ }^{1}$ Stanford University, Stanford, California, USA \\ ${ }^{2}$ Technion - Israel Institute of Technology, Haifa, Israel
}

\begin{abstract}
We consider electrostatic actuators that are pre-energized with charge, which amplifies their response to input voltages. Floatingelectrode actuators were designed, fabricated, and characterized. A non-disruptive measurement of charge is proposed and demonstrated. When the floating electrode is charged, the system has two distinct pull-in voltages. It is shown that the amplitude of charge is proportional to the average of the two pull-in voltages. In this work, we present the correct way of using energy methods to analyze systems that are loaded by both charge and voltage.
\end{abstract}

\section{INTRODUCTION}

In recent years, much attention is given to the effect of dielectric charging in microelectromechanical actuators [1-6]. Proper operation of RF-MEMS capacitive switches requires a predictable and stable pull-in voltage (e.g. [7]), but this voltage is strongly affected by dielectric charging [6]. An even worse effect is a total lock-down of capacitive switches caused by spatial distribution of injected charges [6]. Dielectric charging has adverse effects in other microsystems as, for example, inducing drift in resonators $[8,9]$.

A rigorous analysis of the effect of injected charge on the electromechanical response of electrostatic actuators was presented by Rottenberg et al. [6, 10]. In these investigations, electrostatic forces due to applied voltage and injected charge were derived, and used to solve the equilibrium equation of the system. An alternative approach is using energy methods to derive the system response. The advantage of energy methods is that they can be used to directly compute critical parameters of the dynamic response, without having to integrate the momentum equations in time [11]. However, with reference to dielectric charging, energy methods must be used with care to avoid physically inconsistent results, as explained in this work.

This manuscript has two purposes. The first is to demonstrate the correct way of using energy methods to analyze electrostatic actuators that are subjected to both voltage and charge. The second purpose is to analyze a simple electrostatic actuator that is loaded by voltage and charge, and to show theoretically and experimentally how the charge can be deduced from measured parameters of the electromechanical response.

\section{ANALYSIS}

As a simple model of an electrostatic actuator that is loaded by charge and driven by voltage, we consider the floating-electrode actuator schematically described in Fig. 1. The floating electrode of mass $m$ has a single degree-of-freedom $x$, and is suspended on a linear spring with stiffness $k$. The floating electrode is electrically isolated and may carry a total charge $Q$, and the driving electrode is subjected to a voltage $V$. A fixed parasitic capacitance $C_{p}$ is also considered. Throughout this work, damping is neglected.

The capacitance of the variable driving capacitor is given by

$$
C_{d}=\varepsilon_{0} A /(g-x)
$$

where $\varepsilon_{0}$ is the permittivity of free-space, $A$ is the area of the driving electrode, and $g$ is the gap at the unloaded state (i.e. $V=0$, $Q=0$ ). For brevity, fringing fields are neglected in the analysis.

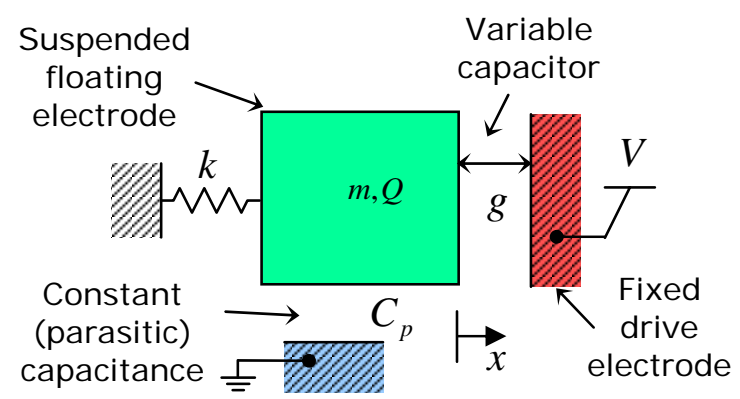

Figure 1: Schematic view of a floating-electrode actuator. The floating electrode with charge $Q$ is suspended on a linear spring and is driven by voltage $V$ on the driving electrode.

When the floating electrode is charged, the driving and parasitic capacitors are effectively connected in parallel. The charge on the driving electrode $q_{d Q}$ and the charge on the groundside of the parasitic capacitance, $q_{p Q}$, are given by

$$
q_{d Q}=-\frac{C_{d} Q}{C_{p}+C_{d}}, \quad q_{p Q}=-\frac{C_{p} Q}{C_{p}+C_{d}}
$$

such that $Q+q_{p Q}+q_{d Q}=0$.

In this actuator, both charge $Q$ and voltage $V$ are the driving parameters of the system. In practice, charge does not change as fast as voltage and accordingly, we assume that the system is first loaded quasi-statically by charge $Q$ while $V=0$, and only then the voltage is applied. This charging may be achieved by connecting the floating electrode to a source with the appropriate voltage. During charging, the suspended floating electrode moves due to the developing electrostatic forces, and reaches an equilibrium state $x=x_{0}$ as derived later on. At this equilibrium state, the charge on the driving electrode reaches the value $q_{d 0}$, which is a function of the charge $Q$

$$
q_{d 0}=-Q \frac{\varepsilon_{0} A}{C_{p}}\left(g+\frac{\varepsilon_{0} A}{C_{p}}-x_{0}\right)^{-1}
$$

Once the floating electrode is charged, the system may be subjected to a voltage $V$. This voltage may be applied gradually so that the loading is quasi-static, or may be applied in a step-function such that a dynamic response is induced. In either case, when a voltage $V$ is applied to the system that was pre-loaded with a charge $Q$, the charge on the driving electrode $q_{d}$ and the charge on the ground-side of the parasitic capacitance $q_{p}$, are given by

$$
q_{d}=\frac{C_{d}\left(V C_{p}-Q\right)}{C_{p}+C_{d}}, \quad q_{p}=-\frac{C_{p}\left(V C_{d}+Q\right)}{C_{p}+C_{d}}
$$

When the system is loaded by voltage, the driving and parasitic capacitances are effectively connected in series. At this state, the electrostatic potential energy $U_{E}$ of the two capacitors is given by

$$
U_{E}=\frac{1}{2} \frac{q_{p}^{2}}{C_{p}}+\frac{1}{2} \frac{q_{d}^{2}}{C_{d}}=\frac{1}{2} \frac{Q^{2}}{C_{p}+C_{d}}+\frac{1}{2} \frac{V^{2}}{\frac{1}{C_{p}}+\frac{1}{C_{d}}}
$$


The mechanical potential $U_{M}$ of the suspension is given by

$$
U_{M}=\frac{1}{2} k x^{2}
$$

To consider the energy of the entire system, we include the potential energy of the voltage source (e.g. battery) [12]. The potential energy of an ideal voltage source is $U_{B}=-q_{B} V$ where $q_{B}$ is the charge that flows from the source to the driving electrode. Accordingly, the potential energy of the voltage source is given by

$$
U_{B}=-\left(q_{d}-q_{d 0}\right) V=\frac{V Q / C_{P}-V^{2}}{\frac{1}{C_{p}}+\frac{1}{C_{d}}}+q_{d 0} V
$$

The total potential of the system is the sum of the mechanical potential, the electrostatic potential, and the potential energy of the voltage source [12]

$$
\begin{aligned}
& \psi(V, Q, x)=U_{M}+U_{E}+U_{B} \\
& =\frac{1}{2} k x^{2}+\frac{1}{2} \frac{Q^{2}}{C_{p}+C_{d}}-\frac{1}{2} \frac{V^{2}}{\frac{1}{C_{p}}+\frac{1}{C_{d}}}+\frac{V Q / C_{P}}{\frac{1}{C_{p}}+\frac{1}{C_{d}}}+q_{d 0} V
\end{aligned}
$$

The careful accounting for the potential energy terms in Eq. (8), especially that of the voltage source in Eq. (7), is essential to correctly account for the electromechanical response of the floating electrode actuator.

\section{Static Response}

Following the methodology in [12], for any given loading $Q$ and $V$, the system may be held at an arbitrary location $x$ by application of a reactive mechanical force. This reactive force is given by the partial derivative of the total potential with respect to the displacement

$$
f=\frac{\partial \psi}{\partial x}=k x-\frac{1}{2} \varepsilon_{0} A\left(V-\frac{Q}{C_{p}}\right)^{2}\left(g+\frac{\varepsilon_{0} A}{C_{p}}-x\right)^{-2}
$$

Equilibrium is a state in which the reactive force vanishes

$$
2 k x=\varepsilon_{0} A\left(V-\frac{Q}{C_{p}}\right)^{2}\left(g+\frac{\varepsilon_{0} A}{C_{p}}-x\right)^{-2}
$$

The stiffness of the system is the partial derivative of the reactive force with respect to the degree of freedom

$$
K(V, Q, x)=\frac{\partial f}{\partial x}=k-\varepsilon_{0} A\left(V-\frac{Q}{C_{p}}\right)^{2}\left(g+\frac{\varepsilon_{0} A}{C_{p}}-x\right)^{-3}
$$

The stability of the system can now be determined by the sign of the stiffness. The system is stable for $K>0$, is unstable for $K<0$ and is critically stable at $x_{c}$ if $K\left(V, Q, x_{c}\right)=0$ and $K\left(V, Q, x_{c}+\delta\right)<0$ where $\delta$ is a small perturbation from $x_{c}$. Stability of an equilibrium state may be determined by substituting (10) into (11) which yields

$$
K_{E q}=k-2 k x\left(g+\frac{\varepsilon_{0} A}{C_{p}}-x\right)^{-1}
$$

Stability of equilibrium is lost (i.e. $K_{E Q}=0$ ) at the pull-in state of the system. It follows that the pull-in displacement is always

$$
x=\frac{1}{3}\left(g+\frac{\varepsilon_{0} A}{C_{p}}\right)
$$

which is compatible with analysis of related systems [12].
The term $V-Q / C_{p}$ in Eqs. (9)-(11) is the effective electrostatic load applied to the suspended electrode, henceforth written as

$$
V^{*}=V-\frac{Q}{C_{p}}
$$

Substituting the displacement (13) back into the equilibrium equation (10), it follows that there are two static pull-in voltages given by

$$
\begin{aligned}
& V_{S P I(I)}=\frac{Q}{C_{p}}+V^{*} S P I \\
& V_{S P I(I I)}=\frac{Q}{C_{p}}-V^{*} S P I
\end{aligned}
$$

where

$$
V_{S P I}^{*}=\sqrt{\frac{8}{27} \frac{k}{\varepsilon_{0} A}}\left(g+\frac{\varepsilon_{0} A}{C_{p}}\right)^{3 / 2}
$$

It is deduced that in the initial loading (i.e. $V=0$ ), static equilibrium is only possible if the charge is within the range

$$
-V^{*} \text { SPI }<\frac{Q}{C_{p}}<V^{*} \text { SPI }
$$

Furthermore, from (15) it follows that the normalized charge is equal to the average of the two pull-in voltages

$$
\frac{Q}{C_{p}}=\frac{1}{2}\left(V_{S P I(I)}+V_{S P I(I I)}\right)
$$

and that the critical effective electrostatic load is given by

$$
V_{S P I}^{*}=\frac{1}{2}\left(V_{S P I(I)}-V_{S P I(I I)}\right)
$$

Therefore, by measuring the positive and negative pull-in voltages (without discharging the floating electrode) we can deduce the amount of charge on the floating electrode. We emphasize that this measurement is insensitive to the mechanical parameters of the system (i.e. mass and stiffness). At the same time, since the system constant $V_{S P I}^{*}$ is independent of charge, we can use property (19) to monitor the quality of measurement.

We may now extract the initial equilibrium displacement resulting form the charge alone (i.e. $V=0$ ), and extract the charge $q_{d 0}$ on the driving electrode in that equilibrium state from (3). Actually, it is simpler to compute $Q$ and $q_{d 0}$ in terms of the equilibrium displacement $x_{0}$ within its stable range $0 \leq x_{0}<\frac{1}{3}\left(g+\varepsilon_{0} A / C_{p}\right)$. Setting $V=0$ in the equilibrium equation (10) and solving for $Q$ yields

$$
\frac{Q}{C_{p}}= \pm \sqrt{2 \frac{k x_{0}}{\varepsilon_{0} A}}\left(g+\frac{\varepsilon_{0} A}{C_{p}}-x_{0}\right)
$$

A typical equilibrium curve of a floating-electrode actuator is schematically illustrated in Fig. 2. This specific equilibrium curve relates to a positive charge $Q>0$. Loading the actuator with charge $Q$ results in the initial displacement $x_{0}$. From this initial state, the system can be subjected to a gradually increasing, positive or negative voltage, until a pull-in instability occurs. In the charged actuator, there are two distinctly different pull-in voltages. For $Q>0$ one pull-in voltage is negative with small amplitude, and the other is positive with large amplitude. In the case $Q=0$, the curve is symmetric in $V$, both positive and negative pull-in voltages have the same amplitude, and in that case we commonly consider only the upper half of the equilibrium curve. 


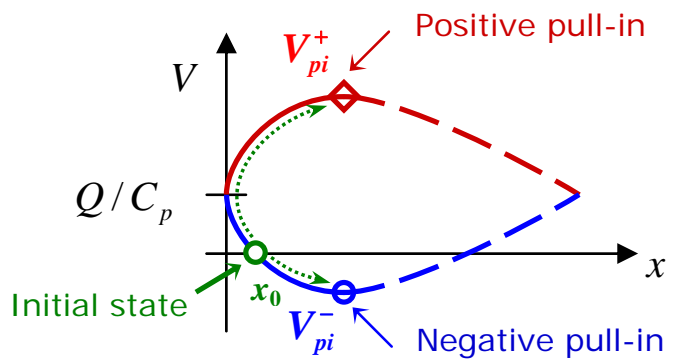

Figure 2: Equilibrium curve of the floating-electrode actuator for a given charge. The charge works like a bias, shifting the curves by $Q / C_{p}$, such that the two pull-in voltages have different amplitudes.

\section{A Modeling Error to be Avoided}

For electrostatic actuators that are driven by voltage only, it turns out that $U_{B}=-2 U_{E}$ (e.g. set $Q=0$ in (5), (7) and (3)). However, it is incorrect to identify the total potential as $\psi^{w}=U_{M}-U_{E}$. In electrostatic actuators that are driven only by voltage this error has no consequence. However, in cases such as the floating-electrode actuator with $Q \neq 0$, the equilibrium equation that is derived using the wrong total potential is given by

$$
2 k x=\varepsilon_{0} A\left(V^{2} Q^{2} / C_{p}{ }^{2}\right)\left(g+\frac{\varepsilon_{0} A}{C_{p}}-x\right)^{-2}
$$

This incorrect result predicts that the equilibrium displacement depends on only the magnitudes of the voltage and charge and not on the their polarities [13, 14], in contrast to Eq. (10) which is verified by experimental results on our actuator.

\section{Dynamic Response}

The location of the suspended floating electrode as function of time can be computed by integrating the momentum equation in time. When the charged actuator is at rest and is subjected to a low step-function voltage, the dynamic response is a periodic vibration, with stagnation states $\dot{x}=0$ at $x>0$. If the step-function voltage is sufficiently high, a non-periodic switching response occurs. The step-function voltage, which is in between the periodic and switching responses, is the dynamic pull-in voltage. By running many such time-integration simulations, we can try to extract the dynamic pull-in voltage, but this is obviously very timeconsuming.

An alternative approach is to use energy methods to extract the dynamic pull-in voltage without any time integration [11, 12].

To this end, we consider the Hamiltonian of the system

$$
H=\frac{1}{2} m \dot{x}^{2}+\psi(V, Q, x)
$$

Immediately after application of the step-function voltage, and before the floating electrode begins to move, the Hamiltonian is equal to the total potential $\psi\left(V, Q, x_{0}\right)$, and assuming there is no damping in the system, from this moment $(t=0)$ the Hamiltonian is constant

$$
H=\frac{1}{2} m \dot{x}^{2}+\psi(V, Q, x)=\psi\left(V, Q, x_{0}\right)
$$

We can now define the dynamic response function which relates the displacement $x$, velocity $\dot{x}$, and applied voltage $V$, at any time $t>0$

$$
D=\frac{1}{2} m \dot{x}^{2}+\psi(V, Q, x)-\psi\left(V, Q, x_{0}\right)
$$

The stagnation states of the system are those states in which velocity vanishes, and are described by the stagnation function

$$
S=\psi(V, Q, x)-\psi\left(V, Q, x_{0}\right)
$$

For any given charge $Q$ and initial displacement $x_{0}$, the stagnation function relates the displacement at stagnation points to the applied step-function voltage. One trivial solution of (25) is $x=x_{0}$ which results from the fact that the initial state is static equilibrium (i.e. $\dot{x}=0$ ) regardless of the step-function voltage $V$ which is about to be applied. By simultaneously solving the equilibrium equation (10) and the stagnation function (25), we may extract the other non-trivial dynamic pull-in displacement and the associated dynamic pull-in voltage

$$
\begin{gathered}
x_{D P I}=\frac{1}{2}\left(g+\frac{\varepsilon_{0} A}{C_{p}}-x_{0}\right) \\
V_{D P I}=\frac{Q}{C_{p}} \pm \frac{1}{2} \sqrt{\frac{k}{\varepsilon_{0} A}}\left(g+\frac{\varepsilon_{0} A}{C_{p}}+x_{0}\right) \sqrt{g+\frac{\varepsilon_{0} A}{C_{p}}-x_{0}}
\end{gathered}
$$

where $Q$ is given by (20).

As in the case of static measurements, the average of the dynamic pull-in is proportional to the charge

$$
\frac{Q}{C_{p}}=\frac{1}{2}\left(V_{D P I(I)}+V_{D P I(I I)}\right)
$$

\section{EXPERIMENT}

Actuators were fabricated using SOI technology (Fig. 3), with a large mass and soft suspension to slow the dynamic response (natural frequency of $f_{n}=268 \mathrm{~Hz}$ ) and thus increase the measurement accuracy.

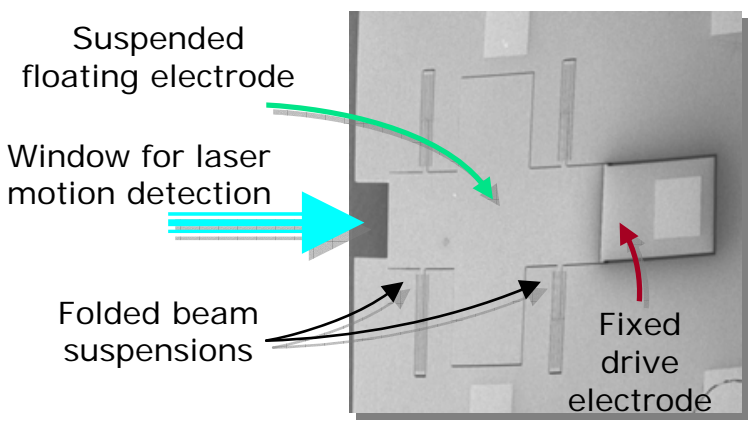

Figure 3: SEM image of floating-electrode actuator. The large rotor plate is suspended on four folded-beam springs. Stoppers that are electrically connected to the floating electrode, prevent contact between the rotor and the fixed driving electrode.

An opening was etched in the device layer to allow accurate measurement of in-plane motion using a Polytec laser vibrometer. The actuator includes stoppers that prevent contact and shorting of the rotor and drive electrode.

The floating electrode is charged by application of voltage through a probe-tip, which is then lifted leaving the charged electrode electrostatically floating. In order to prevent charging of the floating electrode where it faces the driving electrode, the latter is subjected to the same voltage during charging. The parasitic capacitance of the tested actuator is $48.2 \mathrm{pF}$ so when it is charged by a 6Volt source, the total charge is $Q=2.89 \cdot 10^{-10} C=1.8 \cdot 10^{9} \mathrm{e}$. 
Figure 4 presents the measured positive and negative pull-in voltages for various levels of charge. As predicted by the theory, the average of the two pull-in voltages is proportional to the charge. The pull-in voltages were measured a short time after the floating electrode was charged.

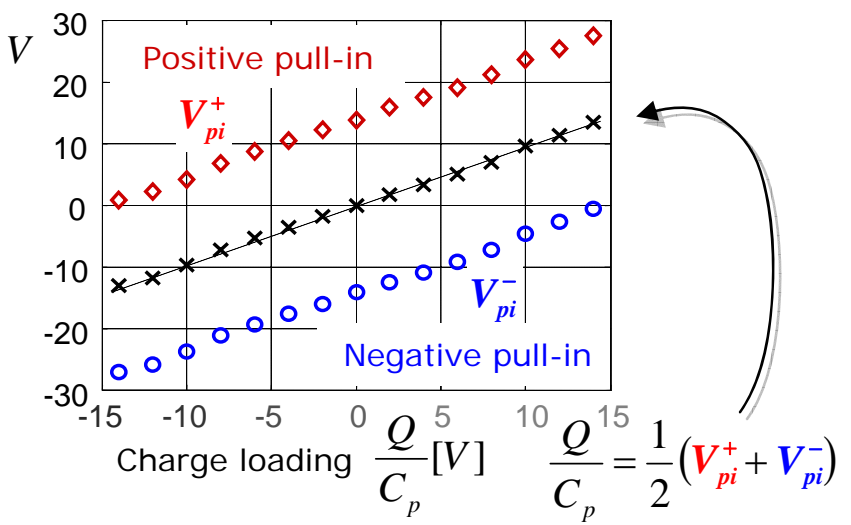

Figure 4: Measured pull-in voltages for different values of charge on the floating electrode. The average of the two pull-in voltages (dashed line) is equal to the charge loading $Q / C_{p}$.
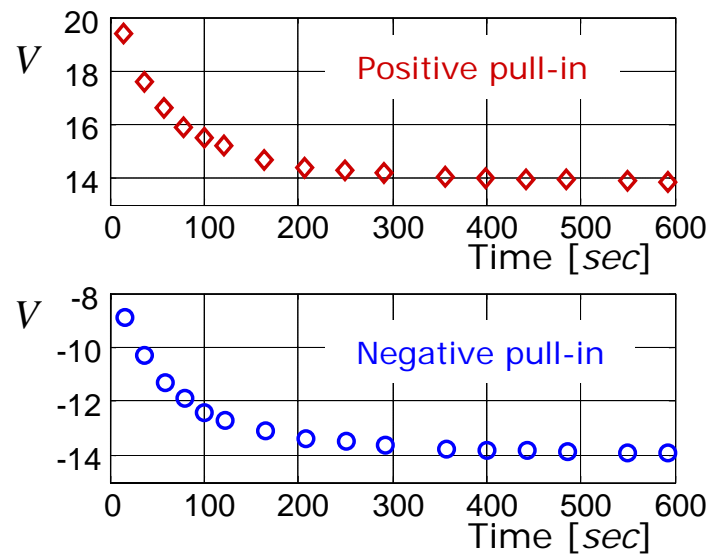

Figure 5: Time decay of positive and negative pull-in voltages. Measurement was performed in ambient conditions of $22^{\circ} \mathrm{C}$ and $50 \%$ relative humidity.

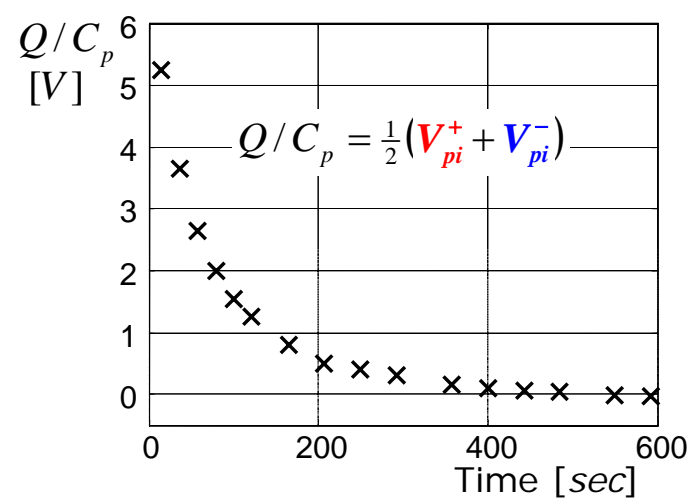

Figure 6: Charge decay as a function of time in the unpackaged actuator. Initial charge was $Q / C_{p}=6$ Volts. The rapid leakage is attributed to high relative humidity.
To measure charge decay, the two pull-in voltages are sequentially and repeatedly measured at fixed time intervals. Figure 5 presents the change in the two pull-in voltages after the floating electrode was charged with a source of 6 Volts. The average of the two, presented in Fig. 6, shows the decay of charge from its initial value. This experiment was performed with an unpackaged device with an ambient relative humidity of $50 \%$. We plan to use our methodology to measure charge and its decay in encapsulated devices, in which pull-in will be identified by the change in capacitance of the driving electrode. It is expected that charge retention will be excellent in a hermetically sealed microshell encapsulation.

\section{ACKNOWLEDGEMENT}

This work was supported by DARPA grant HR0011-06-1-0049 through the Center on Interfacial Engineering in Microelectromechanical Systems (CIEMS), at Stanford University.

\section{REFERENCES}

[1] E.K. Chan, K. Garikipati, and R.W. Dutton, "Characterization of contact electromechanics through capacitance-voltage measurements and simulations," JMEMS, 8, 208-217, 1999.

[2] C. Goldsmith, et al., "Lifetime characterization of capacitive RF MEMS switches," presented at International Microwave Symposium, IEEE MTT-S, 2001.

[3] J.R. Reid, "Simulation and measurement of dielectric charging in electrostatically actuated capacitive microwave switches," MSM 2002, San Juan , Puerto Rico, 2002.

[4] W. M. van Spengen, R. Puers, R. Mertens, and I. De Wolf, "A comprehensive model to predict the charging and reliability of capacitive RF MEMS switches," JMM, 14, 514-521, 2004.

[5] R.W. Herfst, H.G.A. Huizing, P.G. Steeneken, and J. Schmitz, "Characterization of dielectric charging in RF MEMS capacitive switches," IEEE Microelect. Test Structures, 2006.

[6] X. Rottenberg et al. "Analytical model of the DC actuation of electrostatic MEMS devices with distributed dielectric charging and nonplanar electrodes," JMEMS, 16, 1243-1253, 2007.

[7] P. Farinelli et al., "MEMS Switches with Stepped Pull-Down Voltage for Tunable Phase Shifters," MEMSWAVE 2007, Barcelona, 2007.

[8] R. Melamud et al. "Temperature-compensated high-stability silicon resonators," App. Phys. Letters, 90, 244107, 2007.

[9] G. Bahl et al., "Observations of fixed and mobile charge in composite MEMS resonators," Hiton Head workshop, 2008.

[10] X. Rottenberg, B. Nauwelaers, W. De Raedt, and H. A. C. Tilmans, "Distributed dielectric charging and its impact on RF MEMS devices," 34th European Microwave Conf., 2004.

[11] D. Elata and H. Bamberger, "On the dynamic pull-in of electrostatic actuators with multiple degrees of freedom and multiple voltage sources," JMEMS, 15, 131-140, 2006.

[12] D. Elata, "On the static and dynamic response of electrostatic actuators," Bulletin Polish Acad. Sciences, 53, 373-384, 2005.

[13] Y. Nemirovsky et al. "A methodology and model for the pullin parameters of electrostatic actuators," JMEMS, 10, 601615, 2001.

[14] O. Bochobza-Degani et al. "On the effect of residual charges on the pull-in parameters of electrostatic actuators," Sens. \& Act. A, 97(8), 563-568, 2002. 